\title{
Parthénocarpie naturelle chez la tomate. IV. Etude de la parthénocarpie à déterminisme polygénique de la lignée «75/59»
}

\author{
J. Philouze, \\ avec la collaboration technique de M. Milesi
}

INRA, Station d'Amélioration des Plantes maraîchères, Centre de Recherches d'Avignon, F 84140 Montfavet, France

(reçu le 2-5-1988, accepté le 2-10-1988)

Résumé - La lignée «75/59" sélectionnée en Allemagne fédérale à partir du croisement “Atom» x «Bubjekosoko» montre une aptitude à la parthénocarpie naturelle, facultative, de niveau élevé, s'exprimant à toute époque de l'année. Neuf essais ont été réalisés, dans des conditions variées (serre verre ou plastique, chauffée ou froide, champ) à des époques de l'année différentes. Après un traitement avec castration sans pollinisation, les fruits sans graines qui se développent sont d'aspect et de poids comparables à ceux issus de fleurs pollinisées manuellement, quels que soient le type de culture et l'époque de l'année, à l'exception d'un essai où les fruits sont plus petits, ce qui traduit une sensibilité de l'expression de la parthénocarpie aux facteurs du milieu, sans que l'on puisse savoir, dans ce cas précis, quels facteurs du milieu sont en cause.

Des fleurs de «75/59» laissées en fécondation libre donnent en mélange des fruits sans graines (de 34 à $94 \%$ dans nos essais) et des fruits avec graines. Quand il y a ainsi compétition entre fruits avec ou sans graines à l'intérieur d'un bouquet et/ou d'une plante, celle-ci se fait au détriment des fruits sans graines, qui sont alors significativement plus petits que les fruits avec graines issus du même traitement.

La parthénocarpie de «75/59» est récessive. Les tests d'allélisme ont montré qu'elle n'était due ni au gène pat (parthenocarpic) présent chez «Montfavet 191», ni au gène pat-2 de «Severianin». La parthénocarpie de «75/59» serait sous le contrôle de 3 gènes récessifs au moins, et plus probablement 4 ou 5 gènes, dont nous faisons l'hypothèse qu'ils sont indépendants et à action additive. Ces résultats sont discutés et comparés avec ceux obtenus par d'autres auteurs.

Lycopersicon esculentum - tomate - parthénocarpie - parthénocarpie naturelle - déterminisme génétique

Summary - Natural parthenocarpy in tomato. IV. Study of parthenocarpy with polygenic determinism of line "75/59". Line "75/59", bred in West Germany from the cross between "Atom" x "Bubjekosoko", shows an aptitude for high level, natural but facultative parthenocarpy at all times of the year. Nine experiments were carried out under varying conditions (glass or plastic houses, heated or unheated, open field) and at different periods of the year. After treatment involving emasculation without pollination, the appearance and weight of the seedless fruits which developed were comparable to those of the seeded fruits issued from hand-pollinated flowers, regardless of cultivation type or period of year. The findings for one experiment differed, in that the seedless fruits were smaller; this indicates susceptibility of parthenocarpy expression to environmental factors. Notwithstanding, in the present case, it is not possible to identify which environmental factors are involved.

Open pollinated flowers of "75/59" set a mixture of both seedless fruits (from 34 to $94 \%$ in our experiments) and seeded fruits. When both seeded and seedless fruits compete on a single inflorescence and/or plant, the seedless fruits suffer and in this case are significantly smaller than the seeded fruits issued from the same treatment.

The parthenocarpy of "75/59" is recessive. The allelism tests revealed that this is not due to the pat (parthenocarpic) gene present in "Montfavet 191", neither is it due to the pat-2 gene in "Severianin". The parthenocarpy of "75/59" would appear to be controlled by 3 recessive genes at least, and more probably, by 4 or 5 genes. We are of the opinion that these genes act independantly and that their effect is cumulative. These results are discussed and compared to those obtained by other authors.

Lycopersion esculentum - tomato - parthenocarpy - natural parthenocarpy - genetic determinism 


\section{Introduction}

Au cours de l'étude d'une collection variétale (Philouze, 1985), la lignée «75/59» a montré une aptitude de haut niveau à la parthénocarpie, aptitude qui mérite d'être étudiée plus avant. Une revue bibliographique des travaux conduits sur cette lignée et des lignées apparentées ou voisines également sélectionnées en Allemagne fédérale a été faite par Philouze en 1983. Depuis cette date, des travaux réalisés dans différents pays avec la lignée «75/59» ont été publiés.

Nous décrirons ici les principales caractéristiques de «75/59». Puis nous étudierons le comportement de cette lignée dans différentes conditions de milieu (serre verre, tunnel plastique, plein champ) à des époques différentes de l'année. Nous aborderons enfin l'étude génétique du caractère de parthénocarpie de $« 75 / 59 »$, en tentant de déterminer le nombre de gènes en jeu et en faisant les tests d'allélisme avec d'autres origines de parthénocarpie.

\section{Caractéristiques de la lignée «75/59»}

Un véritable travail de sélection pour l'aptitude à la parthénocarpie chez la tomate a été entrepris en Allemagne fédérale avec pour objectif l'obtention de lignées extrêmement précoces. Plusieurs lignées sont issues de ce programme. La lignée $475 / 59$ » semble parmi les plus intéressantes, il s'agit d'une F13 issue du croisement «Atom» $x$ «Bubjekosoko». Des travaux ont été conduits sur ce matériel en Allemagne fédérale, et publiés de 1968 (Reimann-Philipp) à 1978 (Preil); puis les travaux ont cessé, la culture précoce de la tomate sous serre n'étant plus économiquement rentable.

L'aptitude à la parthénocarpie de «75/59» et des autres lignées est héréditaire (Reimann-Philipp et Fuchs, 1969). Selon Preil et Reimann-Philipp (1969), le développement parthénocarpique du fruit ne serait pas dû à une mauvaise qualité du pollen, mais à la production de substances de croissance qui favoriseraient le grossissement de l'ovaire avant que la fécondation n'intervienne (Musehold, 1972). Preil (1973) a montré le rôle des basses températures sur la formation des fruits parthénocarpiques; les fluctuations dans les pourcentages de fruits avec ou sans graines récoltés à un moment donné sont ainsi à mettre en relation avec les fluctuations de températures au moment où les fleurs dont proviennent ces fruits sont au stade de jeunes boutons.

Les plantes de «75/59" sont à croissance déterıminée (gène $s p$ ), avec un développement végétatif limité. Elles portent des fruits petits $(20$ à $30 \mathrm{~g})$, ronds à légèrement ovales, lisses, réguliers, de couleur uniforme avant maturité (gène $u$ ), à 2 ou 3 loges $(2,5$ en moyenne). La mise à fleur est précoce, les fleurs sont grandes et bien formées en toutes saisons, même en jours courts. La maturité des fruits est très précoce. Cette lignée est résistante à Pseudomonas syringae tomato (Okabe), de même que les 2 lignées parentes (Laterrot et Philouze, 1986).

\section{Comportement de «75/59» dans des essais en conditions variées}

Les conditions de milieu autres que les basses températures ont-elles des incidences sur l'expression de la parthénocarpie de «75/59»? Y-a-t-il des conditions dans lesquelles la parthénocarpie de “75/59" s'exprime peu ou mal ? Quelles sont par ailleurs les conditions les plus favorables à la production de graines chez cette lignée ? Des essais conduits dans des conditions de milieu et à des époques de l'année différentes vont nous permettre d'apporter quelques éléments de réponse à ces questions.

\section{Méthodes expérimentales}

Neuf essais, pour lesquels le Tableau I donne le détail des techniques culturales et expérimentales utilisées, ont été conduits en pleine terre dans des conditions de milieu différentes :

- 7 essais ont été conduits sous serre verre : 5 essais au printemps, dont l'un (essai $n^{\circ} 1$ ) en culture antigel, les 4 autres (nos 2, 3, 4 et 5 ) en serre chauffée au cours d'années différentes; et 2 essais à l'automne, en serre chauffée (essais nos 8 et 9 ), au cours d'années différentes;

- 1 essai sous tunnel plastique, au printemps, en culture antigel (essai $n^{\circ} 6$ );

-1 essai au champ (essai $n^{\circ} 7$ ).

Les dates de semis et de plantation sont données dans le Tableau $\mathrm{l}$.

Les traitements suivants ont été appliqués sur les fleurs (Tableau I) :

1) castration des fleurs avant la maturité du pollen, pas de pollinisation;

2) castration des fleurs, pollinisation par du pollen de "Apédice" (lignée riche en pollen de bonne qualité) récolté sur des plantes cultivées dans les mêmes conditions que celles de «75/59" (à l'exception de l'essai $n^{\circ} 1$ où "Apédice" a été cultivée en serre chauffée);

3) castration ou non des fleurs, pollinisation par du pollen de “75/59" récolté sur des plantes cultivées dans les mêmes conditions. Ce traitement correspond donc à une autopollinisation;

4) vibration des inflorescences. Toute inflorescence portant une fleur épanouie est vibrée à l'aide d'un vibreur électrique, à raison de 2 ou 3 passages par semaine;

5) fécondation libre : les fleurs, non castrées, sont laissées libres de s'autopolliniser naturellement.

Ces traitements ont été appliqués sur 1,2 ou 4 plantes, sur 4 bouquets le plus souvent, parfois 5 ou 6 . Les bouquets des traitements 1,2 et 3 sont limités à 8 fleurs au maximum, ceux des traitements 4 et 5 limités à 8 fleurs (essais nos $4,5,6$ ) ou non limités (essais nos 1 , 2,8 et 9 ). 
Tableau I. Techniques culturales et expérimentales pour 9 essais réalisés avec 75/59. Classement des essais d'après la période de castration.

\begin{tabular}{|c|c|c|c|c|c|c|c|c|c|}
\hline Numéro de l'essai & 1 & 2 & 3 & 4 & 5 & 6 & 7 & 8 & 9 \\
\hline & & & \multicolumn{3}{|c|}{ Serre verre } & Tunnel & Champ & \multicolumn{2}{|l|}{ Serre verre } \\
\hline Type de culture & Antigel & & Chauffée & & & Antigel & & Chauffée & \\
\hline \multicolumn{10}{|l|}{ Techniques culturales } \\
\hline $\begin{array}{l}\text { Date semis } \\
\text { Date plantation } \\
\text { Nombre bouquets } \\
\text { Période de castration } \\
\text { (ensemble des bouquets) }\end{array}$ & $\begin{array}{l}22 \text { déc. } \\
10 \text { fév. } \\
4 \\
28 \text { fév. } \rightarrow \\
17 \text { mars }\end{array}$ & $\begin{array}{l}22 \text { déc. } \\
9 \text { fév. } \\
4 \\
\text { (2 mars } \rightarrow \\
15 \text { mars) }\end{array}$ & $\begin{array}{l}29 \text { déc. } \\
16 \text { fév. } \\
4 \\
5 \text { mars } \rightarrow \\
23 \text { mars }\end{array}$ & $\begin{array}{l}29 \text { déc. } \\
15 \text { fév. } \\
4 \\
2 \text { mars } \rightarrow \\
30 \text { mars }\end{array}$ & $\begin{array}{l}13 \text { déc. } \\
31 \text { janv. } \\
4 \\
14 \text { mars } \rightarrow \\
15 \text { avril }\end{array}$ & $\begin{array}{l}21 \text { janv. } \\
11 \text { mars } \\
4 \\
\text { (mi-mars } \rightarrow \\
\text { début avril) }\end{array}$ & $\begin{array}{l}3 \text { mars } \\
22 \text { avril } \\
4 \\
6 \text { mai } \rightarrow \\
27 \text { mai }\end{array}$ & $\begin{array}{l}5 \text { août } \\
7 \text { sept. } \\
5 \\
15 \text { sept. } \rightarrow \\
18 \text { oct. }\end{array}$ & $\begin{array}{l}13 \text { août } \\
16 \text { sept. } \\
6 \\
24 \text { sept. } \rightarrow \\
13 \text { oct. }\end{array}$ \\
\hline \multicolumn{10}{|l|}{ Traitements } \\
\hline $\begin{array}{l}\text { (1) Castration, pas de } \\
\text { pollinisation }\end{array}$ & $+(1) \mathrm{C}$ & - & $+(2) \mathrm{C}$ & $+(2) \mathrm{C}$ & $+(2) \mathrm{C}$ & - & $+(4) \mathrm{C}$ & $+(2) \mathrm{C}$ & $+(1) C$ \\
\hline $\begin{array}{l}\text { (2) Castration, pollinisation } \\
\text { par Apédice }\end{array}$ & $+(1) \mathrm{Ca}$ & - & - & - & $+(2) \mathrm{C}$ & - & $+(2) \mathrm{C}$ & $+(2) C$ & $+(1) C$ \\
\hline $\begin{array}{l}\text { (3) Pollinisation par } 75 / 59 \text {, } \\
\text { avec ou sans castration }\end{array}$ & $+(1) \mathrm{C}$ & - & $+(2) \mathrm{C}$ & $+(2) \mathrm{C}$ & - & - & - & $+(2)$ & $+(1)$ \\
\hline (4) Vibration des inflorescences & $+(1)^{*}$ & $+(2)^{*}$ & - & - & - & - & - & - & $+(1)^{*}$ \\
\hline (5) Fécondation libre & $+(1)^{\star}$ & $+(2)^{*}$ & - & $+(1)$ & $+(2)$ & $+(4)$ & - & $+(2)^{*}$ & $+(1)^{*}$ \\
\hline
\end{tabular}

(n) : nombre de plantes; $\mathrm{C}$ : castration des fleurs; a : pollen venant de plantes cultivées en serre chauffée (dans tous les autres cas, le pollen vient de plantes cultivées dans les mêmes conditions que les plantes de l'essai; " ; bouquets non limités (dans tous les autres cas, les bouquets ont été limités à 8 fleurs maximum).

La période de floraison (de castration) sur l'ensemble des bouquets travaillés, s'étend de début mars à mi-avril pour les essais réalisés sous serre verre et sous tunnel plastique au printemps, en mai pour celui réalisé en champ, de mi-septembre à mi-octobre pour ceux réalisés sous serre verre à l'automne.

Tous les fruits noués ont été récoltés et pesés individuellement, les graines extraites fruit par fruit et comptées. Pour les traitements où le nombre de fleurs a été limité à 8 maximum par bouquet, le pourcentage de nouaison a été calculé. Les comparaisons entre les poids des fruits obtenus à la suite des différents traitements ont été effectuées au moyen du test $t$ de Student. Ces comparaisons ont été faites entre les traitements 1 , 2 et 3 d'une part; d'autre part entre les traitements 4 et 5. De même les comparaisons entre les poids des fruits avec graines ou sans graines à l'intérieur d'un même traitement ont été effectuées au moyen du test $t$ de Student; cependant ce calcul n'a été fait que quand l'une ou l'autre de ces 2 catégories de fruits représentait au moins $9 \%$ du total. Enfin, pour les fruits obtenus à la suite de l'un ou l'autre des traitements avec pollinisation, les coefficients de corrélation entre le poids des fruits et le nombre de graines qu'ils contiennent, ont été calculés.

L'aptitude à la parthénocarpie est jugée selon 2 critères :

- l'aptitude à développer, après castration sans pollinisation, des fruits d'aspect et de taille comparables à ceux obtenus après pollinisation (par «Apédice" ou par «75/59");

- l'aptitude à développer, en fécondation libre, ou éven- tuellement après vibration des inflorescences, un mélange de fruits avec et sans graines.

\section{Résultats et Discussion}

Les résultats sont donnés dans les Tableaux II et III. A la suite du traitement avec castration sans pollinisation, on obtient le plus souvent ( 5 essais sur $7:$ nos 1, 3, 4, 8,9) des fruits dont le poids ne diffère pas significativement de ceux obtenus après un traitement avec pollinisation manuelle par «Apédice» ou «75/59» (Tableau II). Cependant dans un cas (essai $n^{\circ} 7$, au champ), les fruits sont significativement plus gros que ceux obtenus après pollinisation par «Apédice». Dans un autre cas, les fruits sont significativement plus petits que ceux obtenus après pollinisation par "Apédice"; il s'agit de l'essai $n^{\circ} 5$, où la parthénocarpie de «75/59» s'exprime mal à la suite du traitement avec castration sans pollinisation : seulement $45 \%$ des fleurs donnent des fruits, ceux-ci sont très petits $(14,4 \mathrm{~g})$, alors qu'après castration et pollinisation par "Apédice", $93 \%$ des fleurs donnent des fruits de $29,9 \mathrm{~g}$ en moyenne. Le comportement de "75/59" dans cet essai $n^{\circ} 5$ est difficile à interpréter, d'autant plus que dans les essais nos 3 et 4 , réalisés également au printemps sous serre 
Tableau II. Résultats obtenus avec 75/59, après castration sans pollinisation, et après pollinisation par Apédice ou par $75 / 59$.

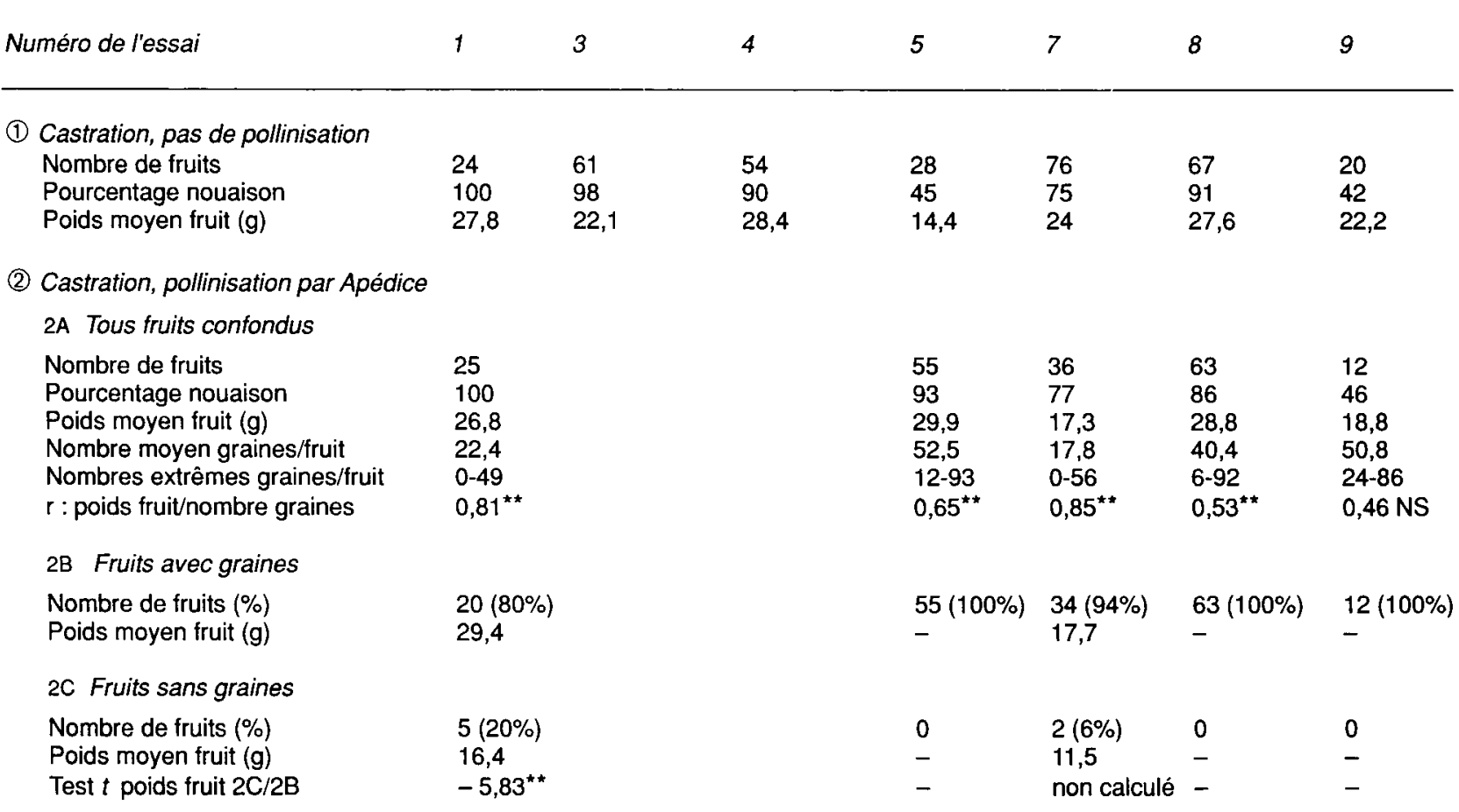

(3) Autopollinisation manuelle

3A Tous fruits confondus

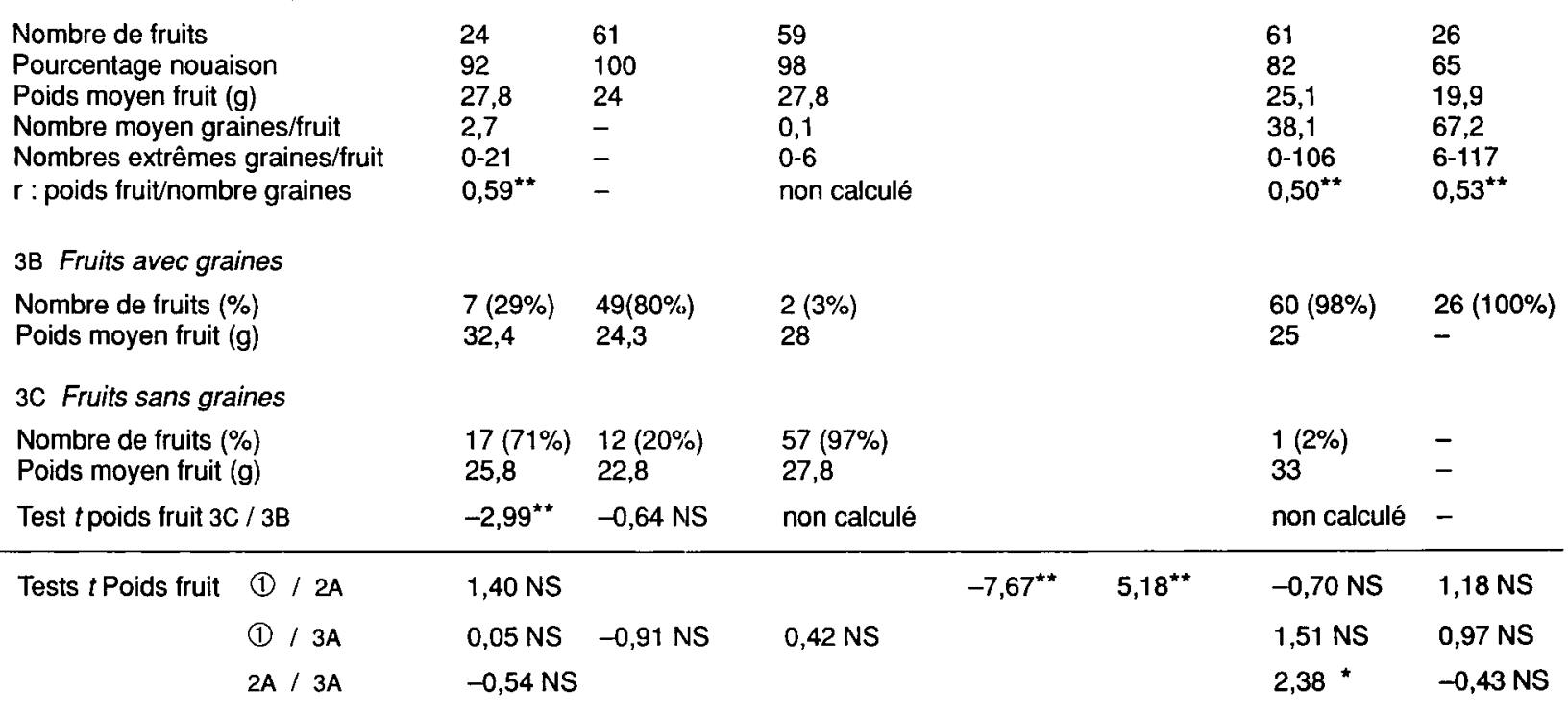

Coefficients de corrélation $\mathrm{r}$ et test $t$ de comparaison de moyennes : NS : non significatif; ${ }^{*}:$ significatif au seuil 0,$01 ;{ }^{* *}$ significatif au seuil 0,01 .

verre chauffée, la parthénocarpie de «75/59" s'exprime très bien à la suite du traitement avec castration sans pollinisation.

Parmi les fruits issus de fleurs castrées pollinisées par "Apédice" (Tableau II), rares sont ceux qui ne contiennent aucune graine (dans ce cas, ces derniers sont nettement plus petits que les fruits avec graines). Ce résultat indique que les gamètes femelles de $« 75 / 59$ " sont parfaitement fonctionnels.

Après autopollinisation manuelle (Tableau II), le pourcentage de fruits sans graines est extrême- 
ment variable selon les essais (de 0 à $97 \%$ pour 5 essais). Si les fleurs de «75/59" ont toujours été bien conformées, il convient de souligner que la quantité de pollen disponible a été très fluctuante d'un essai à l'autre, elle a parfois été quasi nulle. C'est le cas pour l'essai $n^{\circ} 1$, où les basses températures peuvent être incriminées. Dans le cas de l'essai $n^{\circ} 4$, l'absence de pollen, sur des fleurs d'aspect parfaitement normal épanouies en mars, en serre chauffée, n'a pu être expliquée. Notons que dans l'essai $n^{\circ} 3$, conduit aux mêmes dates que l'essai $n^{\circ} 4$, mais une autre année, il y a eu au contraire production de relativement peu de fruits sans graines $(20 \%)$ après autopollinisation ma- nuelle. Enfin dans les essais nos 8 et 9,98 à $100 \%$ des fruits obtenus après autopollinisation manuelle, sans castration, contiennent des graines. Les fleurs épanouies en septembre-octobre contenaient beaucoup de pollen. Ces résultats montrent que la formation du pollen de «75/59 " est particulièrement sensible aux conditions du milieu; c'est avec des fleurs épanouies en début d'automne que la production de graines semble assurée dans les meilleures conditions.

Dans tous les cas où des inflorescences ont été laissées en fécondation libre (Tableau III), il y a production d'un mélange de fruits avec et sans graines; le pourcentage de ces derniers varie de

Tableau III. Résultats obtenus avec 75/59, après vibration des inflorescences ou fécondation libre.

\begin{tabular}{|c|c|c|c|c|c|c|c|}
\hline Numéro de l'essai & 1 & 2 & 4 & 5 & 6 & 8 & 9 \\
\hline \multicolumn{8}{|l|}{ (4) Vibration des inflorescences } \\
\hline \multicolumn{8}{|l|}{ 4A Tous fruits confondus } \\
\hline $\begin{array}{l}\text { Nombre de fruits } \\
\text { Poids moyen fruit }(\mathrm{g}) \\
\text { Nombre moyen graines/fruit } \\
\text { Nombres extrêmes graines/fruit } \\
\mathrm{r} \text { : poids fruit/nombre graines }\end{array}$ & $\begin{array}{l}38 \\
23,6 \\
9,2 \\
0-52 \\
0,80^{* *}\end{array}$ & $\begin{array}{l}55 \\
26,4 \\
51 \\
0-145 \\
0,85^{\star \star}\end{array}$ & & & & & $\begin{array}{l}33 \\
15,8 \\
42,3 \\
0-95 \\
0,36^{\star}\end{array}$ \\
\hline \multicolumn{8}{|l|}{$4 \mathrm{~B}$ Fruits avec graines } \\
\hline $\begin{array}{l}\text { Nombre de fruits }(\%) \\
\text { Poids moyen fruit }(\mathrm{g})\end{array}$ & $\begin{array}{l}18(47 \%) \\
27,8\end{array}$ & $\begin{array}{l}45(82 \%) \\
28,9\end{array}$ & & & & & $\begin{array}{l}30(91 \%) \\
15,5\end{array}$ \\
\hline \multicolumn{8}{|l|}{ 4C Fruits sans graines } \\
\hline $\begin{array}{l}\text { Nombre de fruits }(\%) \\
\text { Poids moyen fruit }(\mathbf{g})\end{array}$ & $\begin{array}{l}20(53 \%) \\
19,8\end{array}$ & $\begin{array}{l}10(18 \%) \\
15\end{array}$ & & & & & $\begin{array}{l}3(9 \%) \\
19\end{array}$ \\
\hline Test $t$ poids fruit $4 \mathrm{C} / 4 \mathrm{~B}$ & $-4,20^{* *}$ & $-3,37^{\star \star}$ & & & & & $0,90 \mathrm{NS}$ \\
\hline \multicolumn{8}{|l|}{ (5) Fécondation libre } \\
\hline \multicolumn{8}{|l|}{ 5A Tous fruits confondus } \\
\hline $\begin{array}{l}\text { Nombre de fruits }(\% \text { nouaison) } \\
\text { Poids moyen fruit }(\mathrm{g}) \\
\text { Nombre moyen graines/fruit } \\
\text { Nombres extrêmes graines/fruit } \\
r: \text { poids fruit/nombre graines }\end{array}$ & $\begin{array}{l}40 \\
16,6 \\
5,3 \\
0-48 \\
0,68^{* *}\end{array}$ & $\begin{array}{l}60 \\
20,9 \\
13,7 \\
0-106 \\
0,72^{\star \star}\end{array}$ & $\begin{array}{l}32(100 \%) \\
18,7 \\
2 \\
0-45 \\
\text { non calculé }\end{array}$ & $\begin{array}{l}49(84 \%) \\
20,9 \\
19,2 \\
0-96 \\
0,77^{\star \star}\end{array}$ & $\begin{array}{l}82(81 \%) \\
19,4 \\
\text { non compté } \\
- \\
-\end{array}$ & $\begin{array}{l}88 \\
22 \\
11,4 \\
0-97 \\
0,27^{\star}\end{array}$ & $\begin{array}{l}32 \\
22,8 \\
38,2 \\
0-133 \\
0,79^{* *}\end{array}$ \\
\hline \multicolumn{8}{|l|}{ 5B Fruits avec graines } \\
\hline $\begin{array}{l}\text { Nombre de fruits (\%) } \\
\text { Poids moyen fruit }(\mathrm{g})\end{array}$ & $\begin{array}{l}12(30 \%) \\
19,9\end{array}$ & $\begin{array}{l}32(53 \%) \\
25\end{array}$ & $\begin{array}{l}3(9 \%) \\
20,3\end{array}$ & $\begin{array}{l}32(65 \%) \\
23,1\end{array}$ & $\begin{array}{l}5(6 \%) \\
21,6\end{array}$ & $\begin{array}{l}50(57 \%) \\
21,1\end{array}$ & $\begin{array}{l}21(66 \%) \\
26\end{array}$ \\
\hline \multicolumn{8}{|l|}{ 5C Fruits sans graines } \\
\hline $\begin{array}{l}\text { Nombre de fruits }(\%) \\
\text { Poids moyen fruit }(\mathrm{g})\end{array}$ & $\begin{array}{l}28(70 \%) \\
15,2\end{array}$ & $\begin{array}{l}28(47 \%) \\
16,1\end{array}$ & $\begin{array}{l}29(91 \%) \\
18,5\end{array}$ & $\begin{array}{l}17(35 \%) \\
16,8\end{array}$ & $\begin{array}{l}77(94 \%) \\
19,3\end{array}$ & $\begin{array}{l}38(43 \%) \\
23,2\end{array}$ & $\begin{array}{l}11(34 \%) \\
16,8\end{array}$ \\
\hline Test $t$ poids fruit $5 \mathrm{C} / 5 \mathrm{~B}$ & $-2,62^{*}$ & $-4,99^{* *}$ & $-0,72$ NS & $-2,59^{*}$ & non calculé & $1,01 \mathrm{NS}$ & $-2,46^{*}$ \\
\hline Test $t$ poids fruit $4 \mathrm{~A} / 5 \mathrm{~A}$ & $4,82^{* *}$ & $2,77^{* *}$ & & & & & $-3,20^{* *}$ \\
\hline
\end{tabular}

Coefficients de corrélation $r$ et tests $t$ de comparaison de moyennes : NS : non significatif; " : significatif au seuil 0,$05 ;{ }^{* *}:$ significatif au seuil 0,0 t. 
34 à 94\%. Dans 6 essais sur 7, les fruits sans graines sont plus petits que les fruits avec graines, les différences sont significatives dans 4 essais. Dans l'essai $n^{\circ} 8$, les fruits sans graines sont légèrement plus gros, mais de façon non significative, que les fruits avec graines. Nos résultats sont en accord avec ceux de Scott et George (1984), qui ont trouvé que les fleurs de "75/59" laissées en fécondation libre donnaient un mélange de fruits avec graines et sans graines : le poids de ces derniers est significativement plus faible que celui des fruits avec graines. Notons que dans l'essai $n^{\circ} 5$, où la parthénocarpie s'est mal exprimée à la suite du traitement avec castration sans pollinisation, $35 \%$ des fruits produits en fécondation libre ne contiennent aucune graine.

La vibration des inflorescences augmente de façon notable la proportion de fruits avec graines (Tableau III). Le poids moyen de ces fruits, par rapport à ceux obtenus en fécondation libre, est significativement plus élevé dans 2 essais $\left(n^{\circ} s 1\right.$ et 2), mais significativement plus faible dans un autre $\left(n^{\circ} 9\right)$. Le nombre de graines par fruit est très augmenté dans l'essai $n^{\circ} 2$, où les conditions sont probablement favorables à la fécondation mais pas à la libération du pollen. Dans l'essai $n^{\circ} 1$, en serre froide, la production de graines, bien qu'améliorée, reste faible. Enfin dans l'essai $n^{\circ} 9$, où les conditions sont favorables à la production de graines, la vibration n'augmente pas de façon significative le nombre de graines par fruit, par rapport aux fruits obtenus sans vibration (environ 40 graines par fruit). De même Scott et George (1984) ont montré, dans un essai conduit au printemps sous abri en conditions non limitantes de températures, que la vibration des inflorescences n'a pas augmenté la production de graines par rapport à la fécondation libre (fruits avec plus de 40 graines en moyenne pour les 2 traitements).

Ces essais montrent par ailleurs que, si les températures froides sont une condition suffisante pour l'apparition de fruits parthénocarpiques (essais $n^{\circ} \mathrm{s} 1$ et 6 ), elles ne sont pas une condition nécessaire, puisque on obtient des fruits sans graines après castration sans pollinisation, et aussi en fécondation libre dans des essais où la température n'est pas un facteur limitant.

II y a une contradiction apparente dans nos résultats : après castration sans pollinisation, il y a le plus souvent formation de fruits sans graines de même poids que les fruits avec graines obtenus après pollinisation par «Apédice» ou «75/59". Mais dans les traitements où la pollinisation intervient (traitements 2, 3, 4,5), les fruits avec graines sont plus gros que les fruits sans graines, dans la grande majorité des cas. Ceci est à mettre en relation avec le fait que, dans tous les cas étudiés (à l'exception du traitement avec pollinisation par “Apédice" dans l'essai $n^{\circ} 9$, où le nombre minimum de graines par fruit est 24 , donc déjà élevé), il y a une corrélation positive entre le poids des fruits et le nombre de graines qu'ils contiennent.

Tout se passe comme si les fruits issus de fleurs castrées non pollinisées se développaient sans compétition sur le bouquet ou sur la plante. Par contre, quand il y a compétition (à l'intérieur du bouquet et/ou de la plante) entre fruits avec graines (ou jeunes ovaires avec ovules fécondés) et fruits sans graines (ou jeunes ovaires avec ovules non fécondés), celle-ci se fait au détriment de ces derniers. Seules des études physiologiques permettraient de comprendre l'origine de cette compétition (différences dans les teneurs en substances de croissance, dans l'aptitude à utiliser les produits de la photosynthèse ?). Musehold (1972) a émis l'hypothèse d'un facteur génétique qui permettrait l'action des substances de croissance chez une lignée parthénocarpique voisine de " $75 / 59$ ", ou qui la bloquerait chez le matériel non parthénocarpique. En tout cas, il semble que la concentration-seuil en substances de croissance nécessaire pour le grossissement du fruit soit disponible chez «75/59», puisque les fleurs castrées non pollinisées donnent des fruits; cette concentration est probablement insuffisante dans l'essai $n^{\circ} 5$, où la parthénocarpie s'exprime mal après le traitement avec castration sans pollinisation.

Cette compétition entre fruits qu'entraine la présence d'ovules fécondés plus ou moins nombreux, a des conséquences pratiques immédiates : en conditions non limitantes pour la pollinisation et la fécondation, il y aura production d'un mélange de fruits avec et sans graines; on introduira donc une hétérogénéité dans le calibre des fruits. II conviendrait par conséquent de se placer dans des conditions où l'on n'obtiendrait que des fruits sans graines : soit en conditions limitantes pour la production de pollen, la pollinisation et la fécondation, par exemple à basse température, soit sur plantes mâles-stériles.

\section{Etude génétique de la parthénocarpie de «75/59»}

L'objectif est d'étudier le déterminisme génétique de la parthénocarpie de «75/59», et de faire les tests d'allélisme avec d'autres origines connues de parthénocarpie. 


\section{Matériel végétal}

Etude du déterminisme génétique. Des croisements ont été réalisés entre «75/59" et 2 lignées non parthénocarpiques :

- «Monalbo». On a observé les lignées parentales, la $F 1$, la $F 2$ et le rétrocroisement de la F1 avec «75/59»;

- «Porvert». Cette lignée mâle-stérile est maintenue par croisement entre plantes soeurs $\mathrm{ms} 1035 \mathrm{aa} / \mathrm{ms} 1035 \mathrm{aa}$ et ms1035aa/++ (le gène aa, anthocyanin absent, est lié au gène de stérilité mâle $m s 1035$ ). On a observé l'hybride F1 entre «75/59" et des plantes mâles-stériles de "Porvert", ainsi que des plantes mâles-stériles de la descendance F2.

Tests d'allélisme. Les tests d'allélisme ont été réalisés entre «75/59» et 2 autres lignées parthénocarpiques :

- “Montfavet 191» porte le gène pat (parthenocarpic) de parthénocarpie (Philouze et Pécaut, 1986). On a observé les lignées parentales et l'hybride $\mathrm{F} 1$;

- «Severianin» porte le gène pat-2 (parthenocarpic-2) (Philouze et Maisonneuve, 1978a). On a observé les lignées parentales, la F1, la F2 et les rétrocroisements de la F1 avec chacun des 2 parents.

\section{Méthodes expérimentales}

Plusieurs essais ont été réalisés, dans des conditions de milieu différentes : serre chauffée en culture de printemps ou d'automne, culture d'été au champ.

Différents traitements ont été appliqués pour apprécier l'aptitude à la parthénocarpie. Dans tous les cas, on a travaillé sur 4 bouquets par plante.

Pour l'étude des hybrides $F \boldsymbol{t}$, en comparaison avec leurs lignées parentales, les 4 bouquets d'une même plante ont subi le même traitement (essai au champ, Tableau IV). Trois traitements différents ont été appliqués : 1) les fleurs ont été castrées mais non pollinisées; 2) les fleurs ont été castrées et pollinisées avec du pollen de «Apédice»; 3 ) les fleurs ont été laissées en fécondation libre. Les bouquets dont les fleurs ont subi les traitements 1 et 2, avec castration, ont été limités à 8 fleurs maximum. Par contre les bouquets laissés en fécondation libre n'ont pas été limités. Les fruits ont été récoltés et pesés individuellement, les fruits des traitements 2 et 3 ont été coupés et notés pour la présence ou l'absence de graines. ères:

L'aptitude à la parthénocarpie est jugée selon 2 cri-

1) l'aptitude à développer, après castration sans pollinisation, des fruits dont l'aspect et le poids, comparés à ceux des fruits obtenus après castration et pollinisation par "Apédice", ne varient pas plus que ceux des témoins parthénocarpiques dans les mêmes conditions;

2) l'aptitude à développer en fécondation libre des fruits sans graines dont le poids, comparé à celui des fruits avec graines, ne varie pas plus que celui des témoins parthénocarpiques dans les mêmes conditions.

L'étude des générations en disjonction, F2 et rétrocroisements, est conduite de manière différente. Dans ce cas, chaque plante doit être appréciée individuellement. Pour cela, le 1er bouquet est soit vibré, soit laissé en fécondation libre, selon les essais; les fleurs des bouquets 2,3 et 4 sont castrées et non pollinisées. Les mêmes traitements sont appliqués aux lignées parentales et aux hybrides $\mathrm{F} 1$, témoins des générations en disjonction. Le nombre de fleurs des fers bouquets n'a pas été limité, celui des bouquets 2,3 et 4 limité à 8 fleurs maximum.

Pour les croisements avec “Monalbo" (3 essais, Tableau V) et "Severianin" (1 essai, Tableau VII), l'appréciation de l'aptitude à la parthénocarpie se fait en comparant, pour chaque plante d'une génération en disjonction, l'aspect et le poids des fruits issus de castration sans pollinisation (bouquets 2,3 et 4 ) à celui des fruits du 1 er bouquet; on effectue ce même rapport de poids de fruits pour chaque plante prise individuellement des témoins parthénocarpiques; on définit comme parthénocarpiques les plantes dont le rapport est égal ou supérieur à celui de la plante témoin la «moins parthénocarpique» (autrement dit chez laquelle ce rapport de poids est le plus faible). De plus, dans les cas où le 1er bouquet a été laissé en fécondation libre, on apprécie l'aptitude à développer des fruits sans graines ayant un poids, par rapport au poids des fruits avec graines, pas plus différent de ce que l'on trouve chez les témoins parthénocarpiques (là encore on utilise les résultats sur plantes individuelles).

Dans le cas du croisement «Porvert» $x$ «75/59" (2 essais, Tableau VI), seules des plantes F2 mâles-stériles ont été plantées. Les fleurs des 4 bouquets étudiés n'ont subi aucun traitement. L'aptitude à la parthénocarpie est jugée sur le développement de fruits sur ces plantes mâles-stériles.

\section{Résultats}

Déterminisme génétique de la parthénocarpie de «75/59»

Croisement avec Monalbo (Tableaux IV et V). La parthénocarpie de «75/59» est récessive : l'hybride F1 «Monalbo" $x$ «75/59" ne montre aucune aptitude à la parthénocarpie (Tableau IV) : après castration sans pollinisation, des fruits très petits se développent, dont le poids ne représente que $26 \%$ de celui des fruits obtenus après pollinisation par "Apédice», alors que chez «75/59" les fruits issus du premier traitement sont plus gros que ceux issus du $2^{e}$ traitement. De plus, tous les fruits obtenus en fécondation libre chez l'hybride contiennent des graines, alors que ce n'est le cas que de $36 \%$ des fruits de $« 75 / 59 »$. Dans cet essai, "Monalbo" (résultats non reportés) n'a pas non plus montré la moindre aptitude à la parthénocarpie. Les fruits à 2 loges de cette lignée pesaient environ $80 \mathrm{~g}$ dans les conditions de l'essai.

Les résultats de 3 essais conduits dans des conditions différentes et portant sur l'étude de la F2 et du rétrocroisement de la F1 avec «75/59» sont donnés dans le Tableau V. Au total une seule plante (essai $n^{\circ} 3$ ) a été notée parthénocarpique 
Tableau IV. Déterminisme génétique de la parthénocarpie de 75/59 et tests d'allélisme. Etude de F1. Essai au champ (plantation 22 avril).

\begin{tabular}{|c|c|c|c|c|c|c|}
\hline \multirow[t]{2}{*}{ Traitement } & \multicolumn{6}{|c|}{ Matériel végétal } \\
\hline & $75 / 59$ & $\begin{array}{l}\text { F1 (Monalbo } \\
x 75 / 59 \text { ) }\end{array}$ & $\begin{array}{l}\text { Montfavet } \\
191\end{array}$ & $\begin{array}{l}F 1(75 / 59 \\
x \text { Montfavet } \\
191)\end{array}$ & Severianin & $\begin{array}{l}F 1(75 / 59 \\
x \text { Severianin })\end{array}$ \\
\hline \multicolumn{7}{|c|}{ (1) Castration, pas de pollinisation } \\
\hline Nombre plantes & 4 & 4 & 3 & 4 & 5 & 4 \\
\hline Nombre fruits & 76 & 85 & 43 & 48 & 34 & \\
\hline Poids moyen fruit $(\mathrm{g})$ & 24 & 11 & 44,7 & 28,8 & 77,2 & 32,6 \\
\hline \multicolumn{7}{|l|}{ (2) Castration, pollinisation par } \\
\hline Nombre plantes & 2 & 1 & & 2 & 5 & 2 \\
\hline \multicolumn{7}{|l|}{ 2A Tous fruits confondus } \\
\hline Nombre fruits & 36 & 22 & & 49 & 41 & 43 \\
\hline Poids moyen fruit (g) & 17,3 & 42,1 & & 47,4 & 91 & 37,4 \\
\hline \multicolumn{7}{|l|}{ 2B Fruits avec graines } \\
\hline Nombre fruits & 34 & 22 & & 49 & 29 & 43 \\
\hline $\begin{array}{l}\text { Pourcentage fruits } \\
\text { Poids moyen fruit }(\mathrm{g})\end{array}$ & $\begin{array}{l}94 \\
17,7\end{array}$ & $\begin{array}{l}100 \\
-\end{array}$ & & $\begin{array}{l}100 \\
-\end{array}$ & $\begin{array}{l}71 \\
90,9\end{array}$ & 100 \\
\hline \multicolumn{7}{|l|}{ 2C Fruits sans graines } \\
\hline Nombre fruits & 2 & 0 & & 0 & 12 & 0 \\
\hline Pourcentage fruits & 6 & 0 & & 0 & 29 & 0 \\
\hline Poids moyen fruit $(\mathrm{g})$ & 11,5 & - & & - & 91,3 & - \\
\hline [Poids (1) / Poids 2A ] $\times 100$ & 139 & 26 & & 61 & 85 & 87 \\
\hline \multicolumn{7}{|l|}{ (3) Fécondation libre } \\
\hline Nombre plantes & 4 & 4 & 2 & 4 & 5 & 4 \\
\hline \multicolumn{7}{|l|}{ 3A Tous fruits confondus } \\
\hline Nombre fruits & 77 & 121 & 50 & 135 & 20 & 107 \\
\hline Poids moyen fruit $(\mathrm{g})$ & 16,2 & 47,1 & 36 & 37,8 & 102,1 & 28,6 \\
\hline \multicolumn{7}{|l|}{ 3B Fruits avec graines } \\
\hline Nombre fruits & 28 & 121 & 0 & 116 & 9 & 102 \\
\hline Pourcentage fruits & & 100 & 0 & 86 & 45 & 95 \\
\hline Poids moyen fruit $(\mathrm{g})$ & (non pesé) & - & - & (non pesé) & 109,9 & 28,5 \\
\hline \multicolumn{7}{|l|}{ 3C Fruits sans graines } \\
\hline Nombre fruits & 49 & 0 & 50 & 19 & 11 & 5 \\
\hline Pourcentage fruits & 64 & 0 & 100 & 14 & 55 & 5 \\
\hline Poids moyen fruit $(\mathrm{g})$ & (non pesé) & - & - & (non pesé) & 95,6 & 31 \\
\hline
\end{tabular}

Les 4 bouquets d'une même plante ont subi le même traitement. Période de castration du 6 mai au 3 juin.

sur les 209 plantes F2 cultivées : le rapport du poids des fruits issus de fleurs castrées non pollinisées par rapport aux fruits obtenus en fécondation libre sur le 1 er bouquet est de $66 \%$; le rapport de poids des fruits sans graines à celui des fruits avec graines est de $99 \%$ chez cette plante, alors que les chiffres les plus faibles obtenus pour “75/59" dans le même essai sont respectivement de $70 \%$ et $83 \%$. De même, sur un total de 105 plantes du rétrocroisement avec «75/59», une seule plante (essai $n^{\circ} 2$ ) a été notée parthénocarpique : le rapport du poids des fruits issus de fleurs castrées non pollinisées à celui des fruits issus de fleurs vibrées est de $56 \%$, alors que le chiffre le plus faible obtenu pour «75/59» dans le même essai est de $52 \%$.

Croisement avec «Porvert» (Tableau VI). L'hybride F1 «Porvert» $x$ «75/59» ne montre aucune aptitude à la parthénocarpie (résultats non 
Tableau V. Déterminisme génétique de la parthénocarpie de 75/59. Etude de générations en ségrégation du croisement (Monalbo x 75/59).

1) Essai sous serre verre chauffée, culture d'automne (semis 12 août, plantation 20 septembre). Bouquet 1 vibré, bouquet 2, 3, 4 : fleurs castrées non pollinisées (du 25 septembre au 21 octobre).

\begin{tabular}{lllll}
\hline \multirow{2}{*}{ Matériel végétal } & \multicolumn{2}{l}{ Nombre de plantes } & \multirow{2}{*}{$a^{*}$} \\
\cline { 2 - 3 } & Total & Normal & Parthéno. \\
\hline $75 / 59$ & 3 & 0 & 3 & $47 ; 52 ; 68$ \\
Monalbo & 3 & 3 & 0 & $($ non pesé) \\
F1 (Monalbo $\times 75 / 59)$ & 3 & 3 & 0 & $14 ; 16 ; 20$ \\
F2 (Monalbo $\times 75 / 59)$ & 50 & 50 & 0 & $(\%$ maxi : 21) \\
BC1 (Monalbo $\times 75 / 59) \times 75 / 59$ & 50 & 50 & 0 & $(\%$ maxi : 46) \\
\hline
\end{tabular}

2) Essai sous serre verre chauffée, culture de printemps (semis 22 décembre, plantation 9 février). Bouquet 1 vibré, bouquets $2,3,4$ : fleurs castrées non pollinisées (du 27 février au 28 mars).

\begin{tabular}{|c|c|c|c|c|}
\hline \multirow[t]{2}{*}{ Matériel végétal } & \multicolumn{3}{|c|}{ Nombre de plantes } & \multirow[t]{2}{*}{$a^{*}$} \\
\hline & Total & Normal & Parthéno. & \\
\hline $75 / 59$ & 2 & 0 & 2 & $52 ; 92$ \\
\hline Monalbo & 2 & 2 & 0 & (non pesé) \\
\hline F1 (Monalbo x 75/59) & 2 & 2 & 0 & (non pesé) \\
\hline F2 (Monalbo x 75/59) & 49 & 49 & 0 & (non pesé) \\
\hline BC1 (Monalbo x 75/59) × 75/59 & 55 & 54 & 1 & (plante parthéno. : 56) \\
\hline
\end{tabular}

3) Essai au champ (semis 2 mars, plantation 24 avril). Bouquet 1 en fécondation libre, bouquets 2, 3, 4 : fleurs castrées non pollinisées (de mi- mai à mi-juin).

\begin{tabular}{|c|c|c|c|c|c|}
\hline \multirow[t]{2}{*}{ Matériel végétal } & \multicolumn{3}{|c|}{ Nombre de plantes } & \multirow[t]{2}{*}{$a^{*}$} & \multirow[t]{2}{*}{$b^{*}$} \\
\hline & Total & Normal & Parthéno. & & \\
\hline $\begin{array}{l}75 / 59 \\
\text { Monalbo } \\
\text { F1 (Monalbo } \times 75 / 59) \\
\text { F2 (Monalbo x 75/59) }\end{array}$ & $\begin{array}{l}2 \\
5 \\
5 \\
110\end{array}$ & $\begin{array}{l}0 \\
5 \\
5 \\
109\end{array}$ & $\begin{array}{l}2 \\
0 \\
0 \\
1\end{array}$ & $\begin{array}{l}70 ; 104 \\
\text { (non pesé) } \\
\text { (non pesé) } \\
\text { (plante parthéno: } 66 \text { ) }\end{array}$ & $\begin{array}{l}83 ; 90 \\
\text { (non pesé) } \\
\text { (non pesé) } \\
\text { (plante parthéno: 99) }\end{array}$ \\
\hline
\end{tabular}

Le premier bouquet a été vibré ou laissé en fécondation libre, le nombre de fleurs non limité. Les fleurs des bouquets 2,3 et 4 ont été castrées et non pollinisées, le nombre de fleurs limité à 8 au maximum.

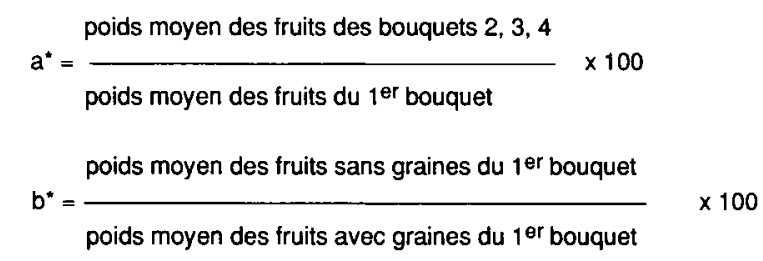

Les chiffres donnés pour $a^{*}$ et $b^{*}$ correspondent à des pourcentages calculés pour des plantes prises individuellement. 
reportés). La F2 a été étudiée en serre verre chauffée, à 2 reprises, l'une au printemps, l'autre à l'automne (Tableau VI). Sur les 312 plantes mâlesstériles cultivées, une seule a développé des fruits à partir de fleurs non pollinisées.

Hypothèses sur le nombre de gènes responsables de la parthénocarpie de "75/59". Les $\chi^{2}$ sont compatibles avec les hypothèses suivantes: 3 gènes minimum dans le cas de la F2 avec "Monalbo"; 3 gènes minimum dans le cas de la F2 avec "Porvert»; 4 gènes minimum si le calcul est fait sur le nombre total de plantes des 2 F2 "Monalbo" $x$ «75/59" et «Porvert" $x$ «75/59"; 5 gènes minimum dans le cas du rétrocroisement («Monalbo" $x$ «75/59") $x$ «75/59".

La seule conclusion que nous puissions donc tirer de ces résultats obtenus par des effectifs limités est que 3 gènes au moins sont responsables de la parthénocarpie de «75/59», et plus vraisernblablement 4 ou 5 .

\section{Tests d'allélisme}

Croisement avec "Montfavet 191" (Tableau IV). Si après castration sans pollinisation on obtient

Tableau VI. Déterminisme génétique de la parthénocarpie de 75/59. Etude de générations en ségrégation du croisement (Porvert x 75/59).

1) Essai sous serre verre chauffée, culture de printemps (semis 29 décembre, plantation 15 février). Bouquets limités à 8 fleurs.

\begin{tabular}{llll}
\hline & \multicolumn{3}{l}{ Nombre de plantes } \\
\cline { 2 - 4 } Matériel végétal & Total & Normal & Parthéno. \\
\hline $\begin{array}{l}\text { F2 (Porvert x 75/59) } \\
\text { plantes [ms 1035 aa] }\end{array}$ & 143 & 142 & 1 \\
\hline
\end{tabular}

2) Essai sous serre verre chauffée, culture d'automne (semis 8 août, plantation 12 septembre). Bouquets non limités.

\section{Nombre de plantes}

\begin{tabular}{llll}
\cline { 2 - 4 } Matériel végétal & Total & Normal & Parthéno. \\
\hline $\begin{array}{l}\text { F2 (Porvert } \times 75 / 59) \\
\text { plantes [ms 1035aa] }\end{array}$ & 169 & 169 & 0 \\
\hline
\end{tabular}

Les 4 bouquets d'une même plante [ $m s 10^{35}$ aa] sont laissés en fécondation libre. chez l'hybride F1 «75/59» x «Montfavet $191 »$ des fruits dont le poids atteint $61 \%$ de celui des fruits développés après castration et pollinisation par "Apédice», seuls $14 \%$ des fruits obtenus en fécondation libre ne renferment pas de graines, alors que pour ce dernier traitement on obtient $100 \%$ des fruits sans graines chez «Montfavet 191 ", et $64 \%$ chez "75/59". Nous en concluons que l'hybride F1 est non parthénocarpique. La parthénocarpie de «75/59" n'est donc pas due au gène pat présent chez «Montfavet 191».

Croisement avec "Severianin". "Severianin» est nettement parthénocarpique (Tableau IV). Les fruits obtenus après castration sans pollinisation ont $85 \%$ du poids des fruits obtenus après pollinisation par "Apédice". De plus $55 \%$ des fruits obtenus en fécondation libre sont parthénocarpiques.

Chez l'hybride F1 «75/59" $x$ "Severianin", seulement $5 \%$ des fruits obtenus en fécondation libre ne renferment aucune graine. Par contre on pourrait déceler une tendance à la parthénocarpie au niveau du développement de fruits après castration sans pollinisation; leur poids représente $87 \%$ de celui des fruits obtenus après pollinisation par «Apédice». Ces fruits obtenus sans pollinisation avaient cependant une allure un peu différente de ceux obtenus après pollinisation, avec en particulier des côtes légèrement marquées, et une maturité assez tardive. Cette légère tendance à la parthénocarpie ne s'est pas retrouvée dans d'autres essais dont les résultats ne sont pas reportés ici. Nous en concluons que la parthénocarpie de “75/59» n'est pas due au gène pat-2 présent chez «Severianin».

La répartition entre plantes normales et parthénocarpiques chez la F2 «75/59" x «Severianin» et les rétrocroisements avec chacun des 2 parents (Tableau VII) a été faite en tenant compte des résultats obtenus avec «75/59" et "Severianin" : on classe comme parthénocarpiques les plantes pour lesquelles le poids des fruits issus de castration sans pollinisation est égal ou supérieur à $52 \%$ (chiffre le plus faible obtenu pour «75/59») du poids des fruits obtenus en fécondation libre. Les $\chi^{2}$ pour la $\mathrm{F} 2$ et le rétrocroisement avec "Severianin" sont en accord avec l'hypothèse d'une ségrégation pour un gène récessif, pat-2 en l'occurence. Le $\chi^{2}$ calculé pour le rétrocroisement avec «75/59" est compatible (à la limite de la signification au seuil 0,05 ) avec l'hypothèse que 3 gènes récessifs au moins sont en jeu. 


\section{Discussion}

D'après nos résultats, la parthénocarpie de «75/59» est récessive; elle serait sous le contrôle de plusieurs gènes, au moins 3 , et plus probablement 4 ou 5 , considérés comme indépendants et à action additive. Ces résultats sont en accord avec nos résultats préliminaires où nous suggérions qu'au moins 3 gènes récessifs étaient en jeu (Philouze et Maisonneuve, 1978c). D'autre part les résultats des tests d'allélisme confirment que la parthénocarpie de «75/59" n'est due ni au gène pat de "Montfavet 191", ni au gène pat-2 de "Severianin" (Philouze et Maisonneuve, 1978b).

La sélection conduite en Allemagne fédérale a permis le cumul dans un même génotype de gènes favorables à la parthénocarpie, gènes qui se trouvaient dispersés chez l'un et l'autre des parents du croisement dont $« 75 / 59 »$ est issu, ou qui sont apparus par mutation au cours de la sélection. En effet, dans un essai au champ dans lequel la parthénocarpie de «75/59" s'est parfaitement exprimée, aucune des 2 variétés «Atom» et «Bubjekosoko" n'a montré la moindre aptitude à la parthénocarpie.

II convient de souligner les difficultés que nous avons eues pour classer en normales et parthénocarpiques les plantes des générations en disjonction : les critères que nous avons retenus pour les plantes parthénocarpiques (aptitude à développer des fruits après castration sans pollinisation, aptitude à développer des fruits sans graines en fécondation libre; comparaison des poids relatifs des fruits ainsi obtenus avec ce que l'on obtient pour les témoins parthénocarpiques) sont en effet quantitatifs, il existe toute une gamme d'intermédiaires, et les chiffres seuils que nous avons retenus sont en fait plus ou moins arbitraires. D'autre part, les comparaisons de poids de fruits obtenus sur des bouquets d'étages différents, surtout si ces bouquets portent des fruits en nombres variables, ne sont guère satisfaisantes. II serait évidemment préférable d'effectuer ces tris dans des conditions de milieu favorables à l'expression de la parthénocarpie, par exemple à basses températures, mais ces conditions sont difficiles ou coûteuses à assurer à Montfavet.

La diversité des variétés normales utilisées en croisement avec $\propto 75 / 59 "$, les différences dans les conditions de milieu, et celles dans les méthodes retenues pour apprécier la parthénocarpie, les difficultés de tri au niveau de plantes individuelles de générations en disjonction, peuvent expliquer les différences entre nos résultats et ceux obtenus par d'autres auteurs.
Pour Vardi (1985) et Vardi et al. (1986), la parthénocarpie de «75/59" serait due à 3 gènes récessifs avec action additive. La lignée «75/59» a été croisée avec une série de lignées comportant des marqueurs génétiques situés sur différents chromosomes. Les essais ont été conduits au champ en hiver, conditions dans lesquelles seuls des fruits sans graines sont produits. L'un des 3 gènes responsables de la parthénocarpie de «75/59» serait sur le chromosome 1 (linkage avec dgt, diageotropica), un autre sur le chromosome 6 (linkage avec $y w$, yellow virescent), le 3 e n'a pas été localisé.

L'étude de croisements entre «75/59» d'une part, et d'autre part 2 lignées (pat-2), "Severianin» (Nuez et al., 1986), et "Par 5-14» à fruits plus petits que "Severianin" (Ferrando et al., 1987), réalisée en culture d'hiver-printemps sous serre plastique non chauffée à Malaga, a conduit ces auteurs à la conclusion que la parthénocarpie de “75/59" était due à 2 gènes récessifs pat-3 et pat-4. Les plantes parthénocarpiques ont l'un ou l'autre des génotypes suivants :

- homozygote pour pat-2 (c'est le cas de "Severianin" et de «PAR 5-14»);

- homozygote pour pat-3 et pat-4 («75/59»);

- homozygote pour l'un ou l'autre des gènes pat-

3 ou pat-4, hétérozygote pour l'autre;

- homozygote pour pat-3 et pat-4+, ou pour pat-3+ et pat-4, et hétérozygote pour pat-2.

Selon ce modèle, les 3 gènes pat-2, pat-3 et pat- 4 ont une récessivité incomplète et une action complémentaire.

Les résultats du croisement entre «75/59» et "Severianin" ne nous permettent pas de confirmer ces hypothèses puisque les disjonctions observées en F2 (Tableau VII) sont en accord avec la disjonction théorique 3 Normaux : 1 Parthénocarpique, pour un gène récessif, et non avec la disjonction théorique 29 Normaux : 35 Parthénocarpiques, qui est celle donnée par le modèle de Nuez et al., (1986). De même les disjonctions théoriques pour le rétrocroisement avec le témoin (pat-2) seraient 1 Normal : 1 Parthénocarpique, et 1 Normal : 3 Parthénocarpiques pour le rétrocroisement avec «75/59».

\section{Conclusion}

La parthénocarpie de “75/59», jugée par l'aptitude à développer des fruits à la suite d'un traitement avec castration sans pollinisation et/ou par l'aptitude à donner un pourcentage non négligeable de 
Tableau VII. Test d'allélisme. Etude de générations en ségrégation du croisement (75/59 x Severianin).

Essai sous serre verre chauffée, culture de printemps (semis 22 décembre, plantation 9 février). Bouquet 1 vibré (nombre de fleurs non limités); bouquets 2, 3, 4 : fleurs castrées non pollinisées (du 24 février au 28 mars) (nombre de fleurs limité à 8 au maximum).

\begin{tabular}{|c|c|c|c|c|c|c|}
\hline \multirow[t]{2}{*}{ Matériel végétal } & \multicolumn{3}{|c|}{ Nombre de plantes } & \multirow[b]{2}{*}{$a^{*}$} & \multicolumn{2}{|c|}{ Calculs de $\chi^{2}$} \\
\hline & Total & Normal & Parthéno. & & Hypothèse & $\chi^{2}$ \\
\hline $75 / 59$ & 2 & 0 & 2 & $52 ; 92$ & & \\
\hline Severianin & 2 & 0 & 2 & $66 ; 91$ & & \\
\hline F1 (75/59 x Severianin) & 2 & 2 & 0 & (non pesé) & & \\
\hline F2 (75/59 x Severianin) & 39 & 34 & 5 & & $3: 1$ & $3,08 \mathrm{NS}$ \\
\hline BC1 $(75 / 59 \times$ Severianin) $\times$ Severianin & 41 & 22 & 19 & & $1: 1$ & 0,22 NS \\
\hline BC1 $(75 / 59 \times$ Severianin) $\times 75 / 59$ & 41 & 40 & 1 & & $7: 1$ & 3,79 NS \\
\hline
\end{tabular}

poids moyen des fruits des bouquets $2,3,4$

$a^{*}=$ poids moyen des fruits du 1 er bouquet

Les chiffres donnés pour $\mathrm{a}^{*}$ correspondent à des pourcentages calculés sur des plantes prises individuellement. Calculs de $\chi^{2}:$ NS : non significatif.

fruits sans graines en fécondation libre, s'est montrée d'un très haut niveau. Elle s'est manifestée à toutes les époques de l'année où nous l'avons testée (printemps, été, automne), en serre ou au champ, en culture chauffée ou froide. Sa mauvaise expression à la suite d'un traitement avec castration sans pollinisation, dans un des 9 essais dont nous donnons les résultats, ne peut pas s'expliquer de façon simple, mais cet exemple nous montre l'importance des facteurs du milieu sur l'expression de la parthénocarpie.

Cette aptitude à la parthénocarpie est facultative. La multiplication de «75/59» ne pose pas de problèmes, à condition de bien choisir l'époque de l'année où on doit l'assurer, de préférence en fin d'été, début d'automne (floraison en septembreoctobre).

La production d'un mélange de fruits plus gros avec graines et de fruits plus petits sans graines, quand les conditions sont favorables à la pollinisation et à la fécondation, représente un handicap pour l'utilisation de la parthénocarpie dans un programme de sélection. Pour surmonter ce handicap, on peut penser à la sélection de génotypes où ces différences de poids entre les 2 types de fruits seraient atténuées, ou bien à la production rendue obligatoire de fruits sans graines, soit par la culture en conditions difficiles, soit par l'association de la parthénocarpie avec des gènes de stérilité mâle par exemple.

Le déterminisme polygénique de la parthénocarpie de $475 / 59$ " la rend peu attractive à utiliser dans un programme de sélection : il est très difficile de récupérer dans les générations en disjonc- tion des plantes d'un niveau de parthénocarpie équivalent à celui de «75/59». En ce qui nous concerne, nous n'avons pas entrepris de travail de sélection d'un matériel parthénocarpique à partir de «75/59», ayant préféré porter nos efforts sur la création d'un matériel (pat-2) à partir de «Severianin». Mais nous savons que la parthénocarpie de "Severianin" s'exprime mal à certaines époques de l'année, en particulier à l'automne (Philouze et Maisonneuve, 1978c); ce qui n'est pas le cas de «75/59» dont la parthénocarpie s'exprime régulièrement tout au long de l'année.

\section{Références}

Ferrando C., Cuartero J., Costa J., Catala M.S. \& Nuez F. (1987) Relationship between the systems pat-2 and pat-3/pat-4 which determine parthenocarpy in tomato. In: Modern Trends in Tomato Genetics and breeding. Synopses 10th Meeting Eucarpia Tomato Working Group, Pontecagnano, Italy, 1987/09/02-06, 8-13

Laterrot H. \& Philouze J. (1986) Sources of resistance to Pseudomonas tomato indicated by Lebaycid treatment. Rep. Tomato Genet. Coop. 36, 23

Musehold J. (1972) Untersuchungen über den Zusammenhang von spontanen Änderungen im Grad der Parthenokarpie und dem natürlichen Wuchsstoffgechalt bei drei Genotypen von Tomaten. Gartenbauwissenschaft 37, 4, 281-292

Nuez F., Costa J. \& Cuartero J. (1986) Genetics of the parthenocarpy for tomato varieties Sub-Arctic Plenty, 75/59 and Severianin. Z. Pflanzenzuchtg 96, 200-206

Philouze J. (1983) Parthénocarpie naturelle chez la tomate. I. Revue bibliographique. Agronomie 3, 7, 611620 
Philouze J. (1985) Parthénocarpie naturelle chez la tomate. II. Etude d'une collection variétale. Agronomie $5,1,47-54$

Philouze J. \& Maisonneuve B. (1978a) Heredity of the natural ability to set parthenocarpic fruits in the Soviet variety Severianin. Rep. Tomato Genet. Coop. 28, 12-13 Philouze J. \& Maisonneuve B. (1978b) Heredity of the natural ability to set parthenocarpic fruits in a German line. Rep. Tomato Genet. Coop. 28, 12

Philouze J. \& Maisonneuve B. (1978c) Breeding tomatoes for their ability to set fruit at low temperatures. In: Genotype and Environment in Glasshouse Tomato Breeding. Proc. Meeting Eucarpia Tomato Working Group, Leningrad, USSR, 1978/05/16-20, 54-64

Philouze J. \& Pécaut P. (1986) Parthénocarpie naturelle chez la tomate. III. Etude de la parthénocarpie due au gène pat (parthenocarpic fruit) de la lignée "Montfavet 191». Agronomie 6, 3, 243-248

Preil W. (1973) Zur Parthenokarpie bei Tomaten in Abhängigkeit vom Temperaturverlauf. Angew. Botanik. 47, $135-140$

Preil W. (1978) Zuchtziele bei Tomaten. Gemüse 14, 2, 48-51

Preil W. \& Reimann-Philipp R. (1969) Untersuchungen über die Einflüsse verschiedener Umweltfaktoren auf die Funktionsfähigkeit der Pollen von Tomaten (Lyco- persicon esculentum Mill.), insbesondere solcher mit erblicher Neigung zur Parthenokarpie. Angew. Botanik 43, 175-193

Reimann-Philipp R. (1968) Die Ausnützung des Merkmals "Parthenokarpie" zur Züchtung extrem frühreifer Sorten bei der Tomate. In: Arbeiten der Abteilung "Höhere Pflanzen". Vorträge gehalten am 4-12-1968 im Max-Planck-Institut für Kulturpflanzenzüchtung, Hamburg, 15-36

Reimann-Philipp R. \& Fuchs G. (1969) Verfrühung der Tomatenernte unter Glas durch Verwendung von zusatzlich und speziell selektierten Genotypen Buschtomaten. Erwerbsgärtner 23, 2153-2155

Scott J.W. \& George W.L. Jr. (1984) Influence of pollination treatments on fruit set and development in parthenocarpic tomato Hortscience 19, 6, 874-876

Vardi E. (1985) Genetics of parthenocarpy in the tomato (Lycopersicon esculentum Mill) lines Severianin and $75 / 59$ and linkage of genes for parthenocarpy with morphological marker genes. M. Sc. Thesis submitted to the Faculty of Agriculture of the Hebrew University of Jerusalem, Rehovot, Israël, p. 101

Vardi E., Lapushner D., \& Frankell R. (1986) Genetics of parthenocarpy in tomato. In: Institute of Field and Garden Crops. Scientific Activities 1982-1985.. Special publication $n^{\circ} 237$. Department of Scientific Publication, the Volcani Center, Bet Dagan, p. 197 